\title{
Effect of Copper Ion Sterilization on Bacterial Community in a Freshwater Recirculating Aquaculture System
}

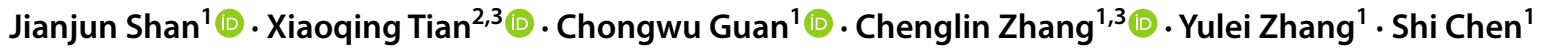

Received: 11 March 2021 / Accepted: 2 November 2021 / Published online: 4 January 2022

(c) The Author(s) 2021

\begin{abstract}
The study aimed to evaluate the safety of copper ion sterilization based on copper ion residues in zebrafish (Brachydanio rerio), as well as bacterial community structure and diversity in recirculating aquaculture systems (RASs). The copper ion content was determined using national food safety standard GB 5009.13-2017. Bacterial community structures and alpha and beta diversity indexes were examined using the 16S rRNA gene sequences produced by Illumina HiSeq sequencing. The results revealed no significant copper ion enrichment in B. rerio when the copper ion concentration was $0.15 \mathrm{mg} / \mathrm{L}$. The relative abundances of Erythrobacter, nitrite bacteria, and Flavanobacteria were clearly higher in the treatment group than in the control and differences in bacterial species richness and diversity were obvious. In addition, there was no sharp decrease in the microflora at the outflow of the copper ion generator. In conjunction with the changes in ammonia nitrogen, nitrate, and nitrite concentrations during the experiment, the results indicated that there were no significant effects on the purification efficacy of the biological filter, but the abundances of beneficial bacteria increased significantly. This is of great relevance in order to understand the response of bacterial communities affected by changing environmental conditions, such as copper ion sterilization.
\end{abstract}

\section{Introduction}

As the aquaculture industry has developed, the number of farmed aquatic species has increased [1]. In addition, improved living standards have allowed consumers to focus on comprehensive nutrition and to demand increasingly higher safety standards in aquaculture production [2]. The health of the aquaculture environment is closely related to the microbial community structure in the water, as well as the levels of nutrients [3]. Microbial community structure,

Jianjun Shan

shanjianjun@fmiri.ac.cn

Chongwu Guan

guanchongwu@fmiri.ac.cn

1 Fishery Machinery and Instrument Research Institute, Chinese Academy of Fishery Sciences, Ministry of Agriculture and Rural of the People's Republic of China, Shanghai 200092, People's Republic of China

2 East China Sea Fisheries Research Institute, Chinese Academy of Fishery Science, Shanghai 200090, People's Republic of China

3 Shanghai Ocean University, Shanghai 201306, People's Republic of China which reflects the biochemical reactions in the ecosystem, can be used as an indicator of the ecological health of the aquaculture environment [4]. Because aquaculture species must be fed constantly, native microorganisms cannot decompose organic matter rapidly enough to prevent water quality deterioration and the growth of harmful microorganisms [5]. The excess undecomposed feed not only pollutes the aquatic environment but also inhibits the respiration of other aquatic animals, possibly even leading to death [6]. High levels of organic matter pollution may also be toxic to humans [7]. However, beneficial microorganisms may play a role in water purification and may serve as food for aquatic organisms $[8,9]$. Therefore, beneficial and harmful aquatic bacteria should be analyzed effectively to ensure the health of aquaculture environments.

Sterilization technology can effectively mitigate the conflict between ecological benefits and economic interests, supporting sustainable development [10]. Copper ion sterilization is an electrochemical sterilization method and copper ions are also one of the most common heavy metal contaminants in water [7]. The author's previous study found that the concentrations of nitrite, nitrate, and total ammonia nitrogen (TAN) and the total number of bacteria remained in a stable state when the concentration of $\mathrm{Cu}^{2+}$ is $0.1-0.2 \mathrm{mg} / \mathrm{L}$ and 
the breeding temperature was $(26 \pm 2)^{\circ} \mathrm{C}$. These conditions were beneficial for the growth of zebrafish (Brachydanio rerio) [7]. However, it is unclear whether $B$. rerio retains copper ions over the long term in aquaculture.

This study thoroughly discusses the safety of the copper ion sterilization process in aquaculture water and provides a theoretical reference for the aquaculture industry with respect to copper ion residue and the microbiome.

\section{Materials and Methods}

The experimental system included a storage tank, a biochemical cotton filter, a moving bed biofilm reactor (MBBR), and a copper ion generator. The filter media parameters were as follows: K5, $\Phi 25^{*} 4 \mathrm{~mm}, 64$ holes, the specific surface area is $800 \mathrm{~m}^{2} / \mathrm{m}^{3}$, the bulk number was $2.1 * 10^{5}$ per $\mathrm{m}^{3}$, the bulk weight was $125 \mathrm{~kg} / \mathrm{m}^{3}$, and the void ratio was $85 \%$. The copper ion generator used was YLD-TLZ-25, Jiangsu Yilida Water Treatment Systems Co., Ltd., China.

Two recirculating aquaculture systems (RASs) for $B$. rerio were constructed. $B$. rerio were acclimated for seven days in an RAS before the experiment began. Fifty healthy $B$. rerio with an average length $2.5-3.0 \mathrm{~cm}$ and a weight of $0.30 \pm 0.05 \mathrm{~g}$ were selected.

The fish were divided into a control group and a treatment group. The control and treatment groups were exposed to $0 \mathrm{mg} / \mathrm{L}$ and $0.15 \mathrm{mg} / \mathrm{L}$ copper ions, respectively. Daily management operations included regular feeding and system sewage treatment. The copper ion concentration was tested three times per week, while TAN, nitrite, nitrate, dissolved oxygen (DO), temperature (T), and $\mathrm{pH}$ were tested daily. Samples of the water and filter material were collected at the end of the experiment. TAN, nitrate, and nitrite concentrations were determined using Nessler's reagent spectrophotometry, zinc-cadmium reduction, and diazotization coupling, respectively. This experiment was performed at the Fisheries Equipment and Engineering research base of the Fishery Machinery and Instrument Research Institute, Chinese Academy of Fisheries Sciences, China in July 2018. The experiment lasted one month.

The RAS water samples $(100 \mathrm{~mL}$ each $)$ and the filter media samples from MBBR (100 g each) were collected in sterile tubes separately and transferred to the laboratory. Samples were collected in triplicate. The filter was washed with sterile fresh water and shaken vigorously to dissolve the biofilm on the carrier. Then, the above solutions were filtered onto $0.22 \mu \mathrm{m}$ filters and frozen at $-80{ }^{\circ} \mathrm{C}$. Ten $B$. rerio each in the treatment group and control group were randomly selected to measure the concentration of copper ion residues in $B$. rerio tissue.

In the treatment group, the biological filter sample was marked $\mathrm{S} 1$, the water sample was marked $\mathrm{S}$, and the water sample from the outlet of the copper ion generator exit was marked $\mathrm{O}$. In the control group, the biological filter sample was marked $\mathrm{N} 1$, and the water sample was marked $\mathrm{N}$.

To maximize read depth for a temporal study of the bacterial communities in the RAS, we used the Illumina HiSeq platform and targeted the V3-V4 region of the $16 \mathrm{~S}$ rRNA gene. The V3-V4 region of the bacterial 16S rRNA gene was amplified using the forward and reverse universal primers 338F (5'-ACTCCTACGGGAGGCAGCA-3') and 806R (5'-GGACTACHVGGGTTCTAAT-3'), respectively. The amplicon mixture was applied to a HiSeq $2500 \mathrm{MiSeq}$ Genome Sequencer (Illumina, San Diego, CA, USA). Bacterial community composition and biodiversity were determined by Shanghai Majorbio Bio-Pharm Technology Co., Ltd. (Shanghai, China).

\section{Data Analysis}

The extracted high-quality sequences were aligned using PyNAST and UCLUST. The unique sequences were classified into operational taxonomic units (OTUs) at a threshold of $97 \%$ identity using UCLUST. Chimera Slayer was used to remove potential chimeric sequences from the set of representative OTUs. MOTHUR was used for data analysis. The data were analyzed on the free online platform of Majorbio Cloud Platform (www.majorbio.com). Statistical analyses were performed using SPSS V.17.0 [11]. The mean (M) and standard deviation (SD) were used to explore the relationship between specific explanatory variables and outcome variables for water quality results and concentration of copper ions in $B$. rerio after exposure to $0.15 \mathrm{mg} / \mathrm{L}$ copper ions.

\section{NCBI Sequence Accession Numbers}

Bacterial V3-V4 16S rRNA gene sequences generated in this study are available from the NCBI SRA (SRP297447).

\section{Results}

\section{RAS Water Quality Parameters}

The concentrations of TAN, nitrate, and nitrite in the test group were $0.04-0.35 \mathrm{mg} / \mathrm{L}, 0.04-0.01 \mathrm{mg} / \mathrm{L}$, and $0.03-0.01 \mathrm{mg} / \mathrm{L}$, respectively, with averages of $0.13 \pm 0.08 \mathrm{mg} / \mathrm{L}, 0.02 \pm 0.01 \mathrm{mg} / \mathrm{L}$, and $0.02 \pm 0.01 \mathrm{mg} / \mathrm{L}$, respectively. In the control group, the concentrations of TAN, nitrate, and nitrite were $0.05-0.26 \mathrm{mg} / \mathrm{L}, 0.18-0.01 \mathrm{mg} / \mathrm{L}$, and $0.08-0.02 \mathrm{mg} / \mathrm{L}$, respectively, with averages of $0.14 \pm 0.07 \mathrm{mg} / \mathrm{L}, 0.05 \pm 0.04 \mathrm{mg} / \mathrm{L}$, and $0.03 \pm 0.02 \mathrm{mg} / \mathrm{L}$, respectively (Fig. 1). 
Fig. 1 The results of water quality. *In this figure, the treatment groups were marked $\mathrm{T}$ - and the control groups were marked C-.The concentrations of TAN, nitrate, and nitrite were $0.13 \pm 0.08 \mathrm{mg} / \mathrm{l}$, $0.02 \pm 0.01 \mathrm{mg} / \mathrm{l}$, and $0.02 \pm 0.01 \mathrm{mg} / \mathrm{l}$ in the test group and $0.14 \pm 0.07 \mathrm{mg} / \mathrm{l}$, $0.05 \pm 0.04 \mathrm{mg} / \mathrm{l}$, and $0.03 \pm 0.02 \mathrm{mg} / \mathrm{l}$ in the control group

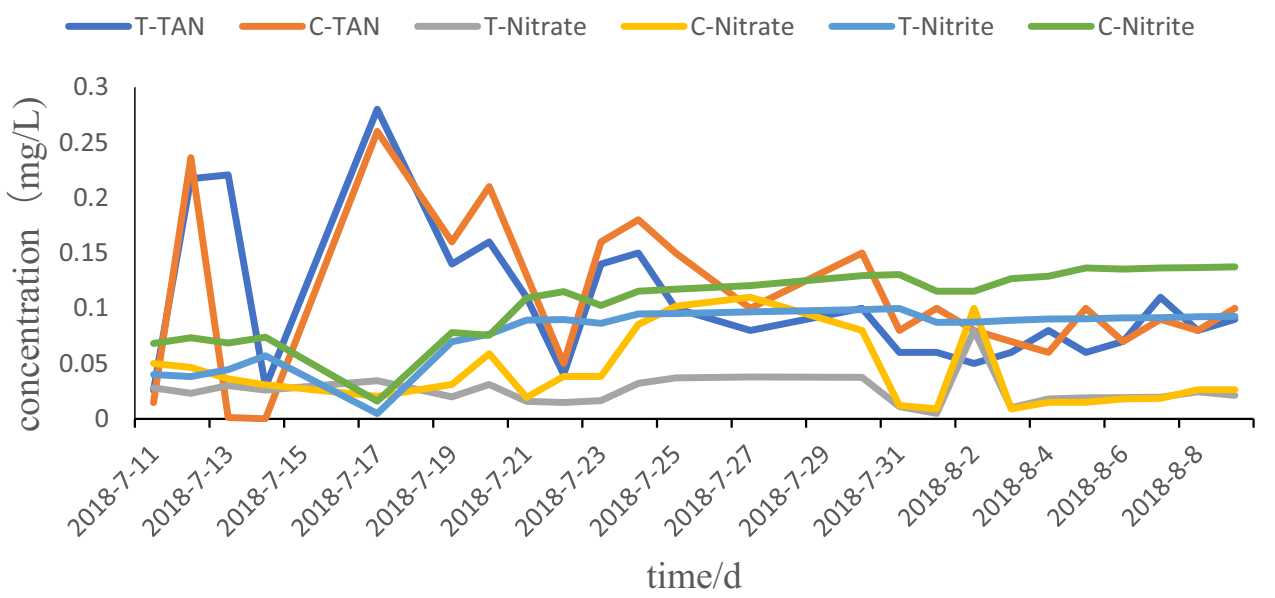

\section{Residual Copper lons in B. rerio}

B. rerio were sampled one day after the last feeding. Selecting some whole fish sample using graphite furnace atomic absorption spectrometry (NHC 2017) detect the copper ion content, respectively. The average values of the cupric ion concentrations in the treatment and control groups were both $16.3 \mathrm{mg} / \mathrm{kg}$.

\section{Influence of Copper Ions on the Microbiology of the RAS}

Based on homologous sequence alignments and clustering, using the information extracted from the RDP and BLAST databases, the OTUs were taxonomically identified to the lowest level possible. The Shannon diversity curves for those samples reached the saturation phase, indicating that the majority of the bacterial phylotypes in the sample had been identified (Supp. Fig. I).

Alpha-diversity data for the samples generated by highthroughput sequencing are shown in Supp. Table1, including the Shannon, Simpson, Chao, and Coverage indexes. The estimated sample coverage (Good's coverage) was about $99 \%$, which indicated that sequencing accuracy and reprehensibility was acceptable. No significant difference in estimated OTU richness (Chao) was observed between the two groups.

However, significant differences in estimations of community diversity between the two groups were identified, including the Shannon index at $4.62 \pm 0.16$ in the treatment group vs. $5.15 \pm 0.13$ in the control, with $\mathrm{P}=0.01$ and $4.34 \pm 0.25$ in the treatment group vs. $4.96 \pm 0.08$ in the control, with $\mathrm{P}=0.04$ for the filter and water samples, respectively, indicating that microbiota diversity was greater in the treatment groups. In addition, there were no significant differences in estimations of community diversity between the $\mathrm{S}$ and the $\mathrm{O}$ in the treatment groups (Shannon $4.34 \pm 0.25$ vs. $4.07 \pm 0.17, P=0.2$ ) (Supp. Tab.1 and Fig. 2).

Bacterial differences were further investigated. Principal co-ordinates analysis (PCoA) of the sequencing data identified differences between the treatment-group clusters and the control-group clusters, with the following main principal component (PC) scores: PC1 was $48.81 \%$ and PC2 was $26.16 \%$. These PC scores demonstrated that the clustering pattern differed among groups (Fig. 3). N and N1 were in the same area and were distinct from $\mathrm{S}, \mathrm{S} 1$, and $\mathrm{O}$, which indicated that bacterial community structure differed between the treatment group and the control group. This suggested that copper ion sterilization had a significant effect in the RAC.

Welch' s t-test for shannon index

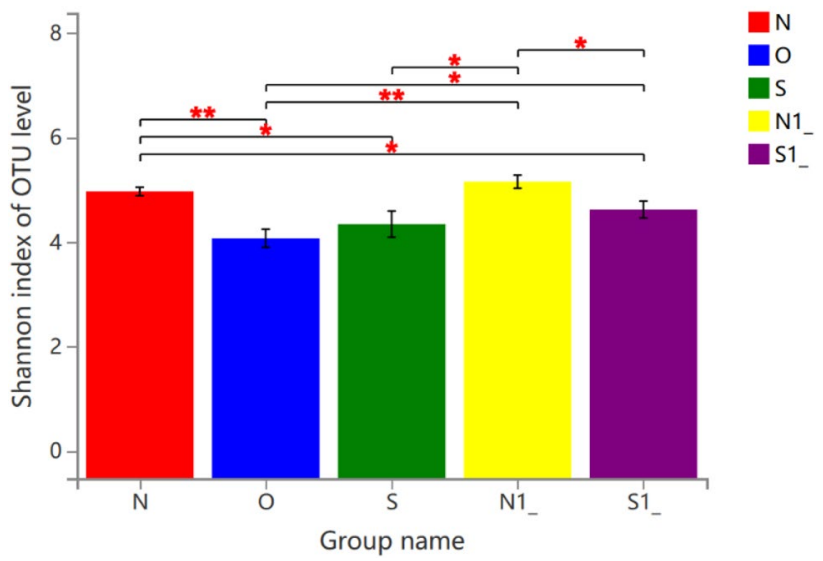

Fig. 2 Microbial diversity among groups, as measured by Shannon index. *Data are expressed as mean \pm standard deviation (S.D.) for each group $(n=3)$. $* P<0.05, * * P<0.01, * * * P<0.001$. Error bars show the standard error of mean (S.E.M.), and the $P$-values are from two-tailed Student's $t$ test 


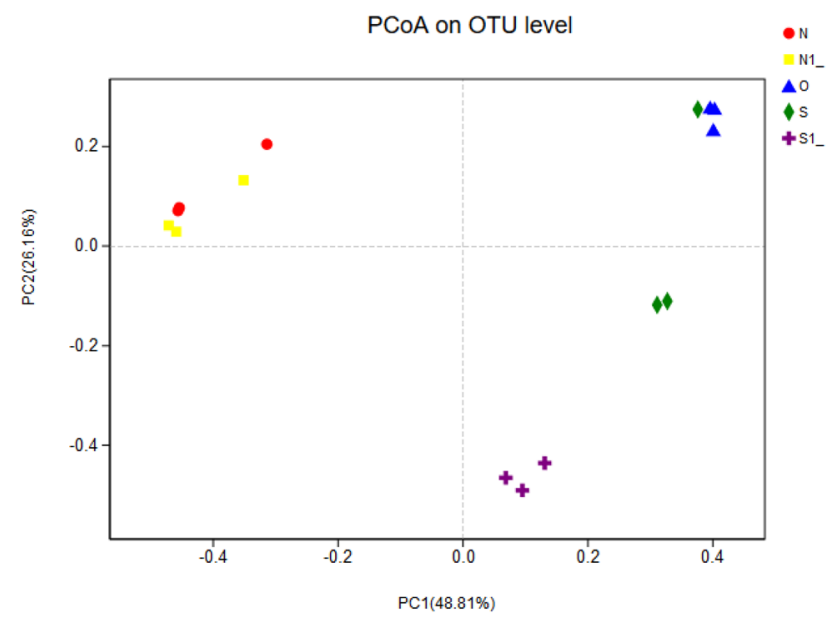

Fig. 3 Principal coordinate analysis plots. *showing the microbiota in the treatment groups and the control groups ( $n=3$ per group). Principal co-ordinates analysis ( $\mathrm{PCoA})$ of the sequencing data identified differences between the treatment-group clusters and the controlgroup clusters, with the following main principal component (PC) scores: PC1 was $48.81 \%$ and PC2 was $26.16 \%$

The species abundance of each sample was calculated at different taxonomic levels by community composition analysis. At the phylum level, the relative abundance of the Proteobacteria was high in filter material from both the control group and the treatment group, accounting for $31.6 \%$ and $42.4 \%$, respectively (Fig. 4). The five mostabundant bacterial classes in the control group were Actinobacteria (20.04\%), Gammaproteobacteria (17.88\%), Alphaproteobacteria (14.82\%), Caldilineae (8.14\%), and Deltaproteobacteria (4.86\%), while the five most-abundant bacterial classes in the treatment group were Alphaproteobacteria (26.65\%), Actinobacteria (13.34\%), Flavobacteria (11.59\%), Gammaproteobacteria $(9.0 \%)$, and Thermomicrobia $(8.81 \%)$.
There were differences in the water samples between the treatment group and the control group. The dominant phylum in the control group was Actinomycetes, accounting for $40.10 \%$, while the dominant phylum in the treatment group was Bacteroidetes, accounting for $38.90 \%$ in the S and $48.80 \%$ in the $\mathrm{O}$ (Fig. 4). However, the communities of low-abundance microbes were similar between the $\mathrm{S}$ and $\mathrm{O}$ in the treatment group, indicating that the aquatic environment was homogeneous.

Species difference analysis revealed significant differences between groups by Welch's $t$ test. There were differences in the microflora genera between the treatment group and the control group. The genera that were significantly different in the filter included Mycobacterium, Rhodobacteraceae, Defluviimonas, Flavobacterium, Nitrosomonas, Mesorhizobium, and Ruegeria and the overwhelmingly dominant genus was norank_f_JTB255_marine_benthic_ group. However, in water, NS3a_marine_group, norank_f JTB255_marine_benthic_group, Candidatus_Aquiluna, unclassified_f_Microbacteriaceae, and Ruegeria were significantly different and the overwhelmingly dominant genus was Robiginitalea. Both norank_f_JTB255_marine_benthic_group and Ruegeria were more abundant in the control group than in the treatment group. In addition, there were significant differences in the abundance of the same strain between the water samples and filter materials (Fig. 5).

In the treatment group, only Candidatus_Aquiluna and norank_f_Saprospiraceae were significantly different between the $\mathrm{S}$ and $\mathrm{O}$. In the control group, norank $f \_C a l d i$ lineaceae and norank_f_Saprospiraceae were detected on the filter, while norank_f_Saprospiraceae, Haloferula, and norank $f_{-} \_$Cryomorphaceae were detected in the water (Fig. 5).

The relative abundances of Erythrobacter, nitrite bacteria, and Flavanobacteria were greater in the treatment group than in the control group. These microorganism were also
Fig. 4 Analysis of Bacterial community composition in the filter and water samples on the phylum level. *The five mostabundant bacterial classes in the control group were Actinobacteria $(20.04 \%)$, Gammaproteobacteria (17.88\%), Alphaproteobacteria (14.82\%), Caldilineae $(8.14 \%)$, and Deltaproteobacteria $(4.86 \%)$, while the five most-abundant bacterial classes in the treatment group were Alphaproteobacteria (26.65\%), Actinobacteria (13.34\%), Flavobacteria (11.59\%), Gammaproteobacteria $(9.0 \%)$, and Thermomicrobia $(8.81 \%)$

\section{Classification of phylum about bacteria}

$\square \mathrm{S} 1 \square \mathrm{N} 1 \square \mathrm{S} \backsim \mathrm{N}=\mathrm{O}$

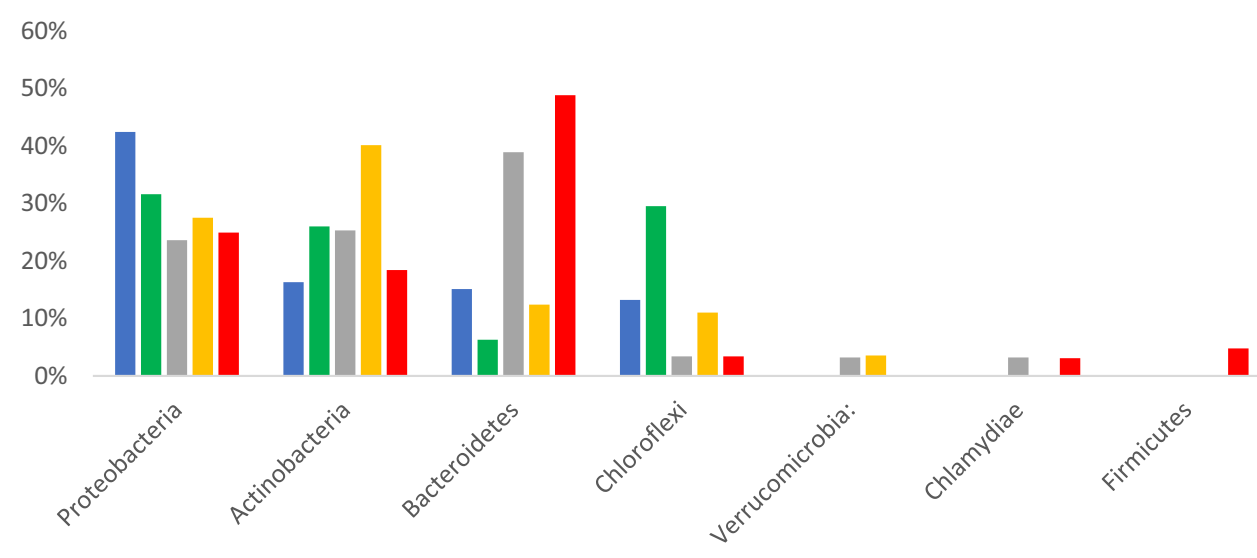


Fig. 5 Significant differences in bacterial community composition between the treatment group and the control groups at the genus level. *Statistical analysis was performed using Welch's $t$ test ( $n=3$ per group). $* P<0.05$, ** $P<0.001$; treatment group vs. control group

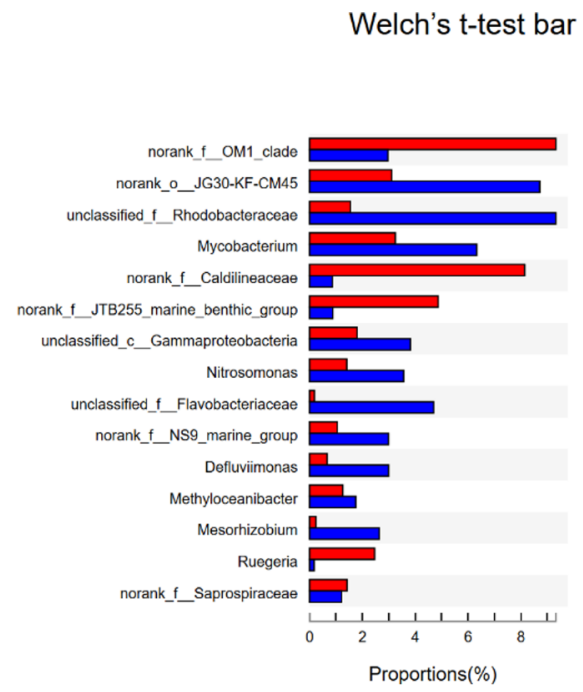

$\square \mathrm{N} 1$

as 1

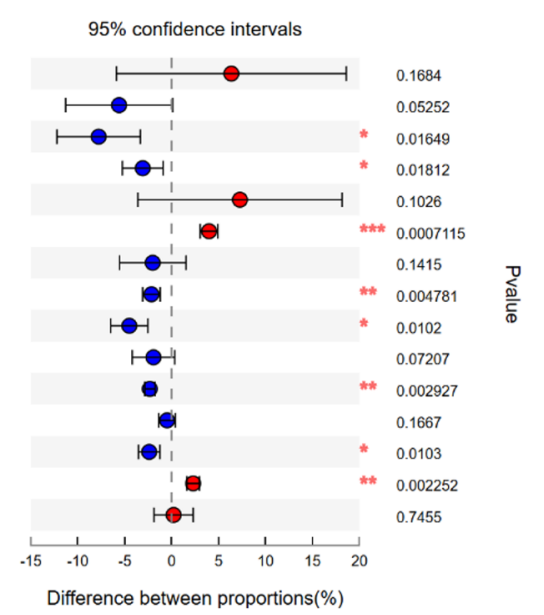

a

Welch's t-test bar plot on Genus level

$95 \%$ confidence intervals
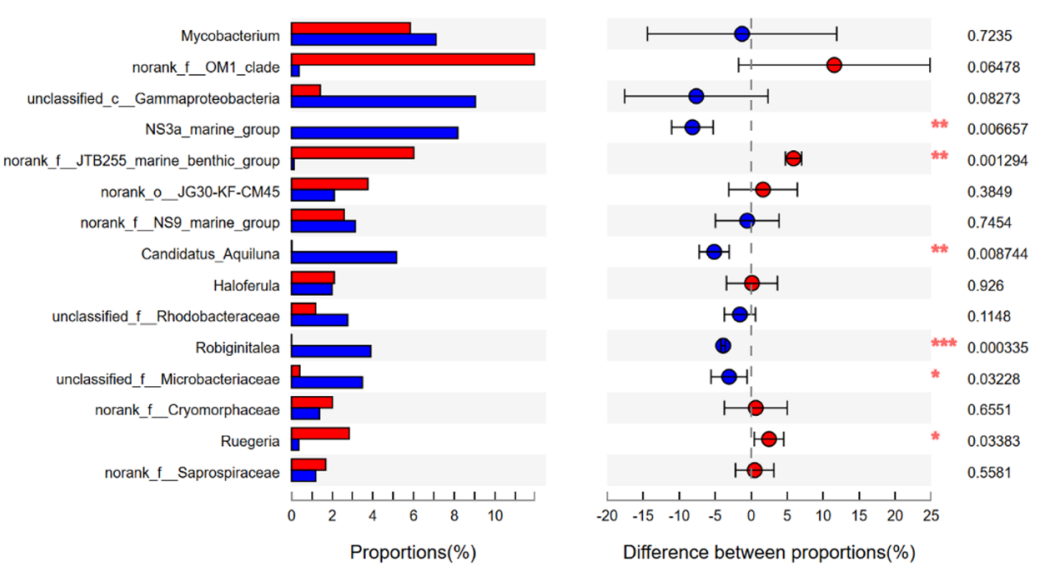

ํㅣㅁ

Welch's t-test bar plot on Genus level
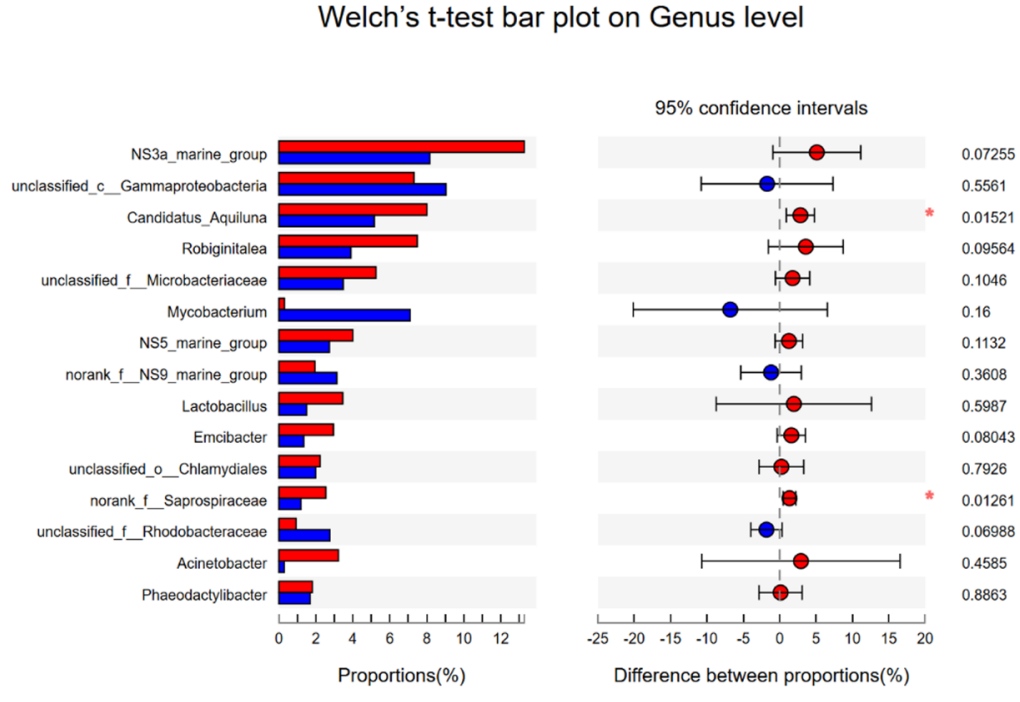

$\square 0$ 
most abundant in the sewage treatment and nitrogen removal systems in the biofilm reactor.

\section{Discussion}

Various factors affect the survival of aquatic economic animals in RASs, including differences in system scale, water properties, and microbial community composition. Suitable water conditions are essential for the survival of aquatic economic animals, such as fish. In this experiment, there were no obvious changes in TAN, nitrate, and nitrite when the copper ions were $0.15 \mathrm{mg} / \mathrm{L}$. The average values of the tested water properties did not reach harmful levels that would negatively impact aquaculture organisms [7]. In addition, there was no significant enrichment of copper ions in $B$. rerio when the copper ions were $0.15 \mathrm{mg} / \mathrm{L}$.

Microbial ecological theory in aquaculture has the potential to extend RAS capabilities [12]. Further identifying the interactions between microorganisms and system design could alleviate concerns regarding the sustainability of aquaculture. The results of Bartelme et al. [13] studied the bacterial and archaeal community structure of a commercial-scale freshwater RAS raising Perca flavescens (yellow perch) and found that $>99.9 \%$ of the archaeal 16S rRNA gene sequences were classified to a single taxon associated with known ammonia-oxidizing archaea. Therefore, archaea will be focused on in a spatial study related to nitrification in the future.

It is likely true that each microbial community assemblage will be unique among RASs, i.e., each RAS has a unique "microbial fingerprint" [13]. The Shannon index, Simpson diversity index, Chao1, and observed species in each sample were used to evaluate species richness and diversity. The results indicated that bacterial species richness and community diversity varied distinctly among the samples. There were differences in the microflora genera between the treatment group and the control group, especially within Rhodobacteraceae, Flavobacteriaceae, Haloferula, and nitrite bacteria.

Rhodobacteraceae unclassified belongs to the family Rhodobacteraceae, in the Alphaproteobacteria, and is a purple non-sulfur bacteria [14]. Depending on extracellular enzymes, heterotrophic metabolic reactions can be carried out by utilizing a variety of organic carbon sources in aquaculture water [14]. In anaerobic or hypoxic conditions, Rhodobacteraceae effectively absorbs phosphate, reduces the phosphorus load in high-density aquaculture, and can play a role in reducing the chemical oxygen demand of water [15]. Flavobacteriaceae unclassified, which belongs to the Flavobacteriaceae (Bacteroidetes), is a facultative anaerobic bacterium. This bacterium performs dissimilatory nitrate reduction [16], reflecting the denitrification process in the filter. In addition, Flavobacterium psychrophilum, belonging to the Flavobacteriaceae, has been found to be a harmful microorganism. Flavobacterium psychrophilum is a gramnegative, filamentous, psychrotrophic bacterium, and is the causative agent of bacterial cold-water disease and rainbow trout syndrome in freshwater salmonid fish worldwide, generating injuries and high mortality rates [17]. Therefore, more attention should be paid to this genus needs in the future. Haloferula belongs to the Chloroflexi and is also a facultative anaerobic microorganism. This taxon performs photosynthesis that neither produces oxygen nor fixes nitrogen [14]. Ammonia-oxidizing bacteria (AOB; nitrite bacteria) and nitrite-oxidizing bacteria (NOB; nitrite bacteria) performed nitrification in the biological filter in this study. Nitrifying and denitrifying microorganisms can greatly shorten the nitrogen removal time in biological filters and improve nitrogen removal efficiency [18-21]. Nitrosomonas was the main nitrifying bacteria in the treatment group. Lactobacillus, a beneficial microorganism [22], was detected in water samples from the outlet of the copper ion generator. Methyloceanibacter and Mesorhizobium were detected in the filter. Some studies [23] have found that adding Rhizobium to the fodder of Litopenaeus vannamei reduced the feed coefficient and improved shrimp growth, survival rate, body condition, specific growth rate, antioxidant enzyme activity, and disease resistance.

The results showed that the relative abundances of Erythrobacter, nitrite bacteria, and Flavanobacteria were higher in the treatment group than in the control group. These microorganisms were also the most abundant in the sewage treatment and nitrogen removal systems in the biofilm reactor. In addition, Nitrosomonas was the main nitrifying bacteria. Lactobacillus was detected in the water samples from the outlet of the copper ion generator. Methyloceanibacter and Mesorhizobium were detected in the filter. These microorganisms improve the environmental quality of aquaculture water and provide a foundation for efficient, highyield aquaculture [24].

However, norank_f_caldilineaceae was detected in the filter of the control group. This taxon [25] is not conducive to the purification of aquaculture water and sometimes contains opportunistic pathogens and other commercially detrimental organisms in RASs which is similar to the results of Bartelme et al. [13].

Copper ion sterilization indirectly enriched the beneficial bacteria, which supported the production of a variety of digestive enzymes in a specific environment, promoted the digestion and absorption of nutrients, and improved the feed utilization rate.

Above all, in addition to characterizing the numbers and species of microorganisms in samples, it is important to collect data on their physiological states [26]. The method of bacterial community analysis used in this study provided 
only limited information on the activities and physiological states of microorganisms. It is necessary to combine metagenomic, single-cell microbiology to supplement and improve this study. For example, viability PCR could be used in future research to observe the activity levels of Rhodobacteraceae, Flavobacteriaceae, Haloferula, and nitrite bacteria.

In addition, the phenotypes and genotypes of microorganisms respond to different physicochemical stressors, including germicidal UV light and antimicrobials. Muñoz et al. studied the resistance, phenotype, and molecular response of Lactobacillus to different physicochemical stressors and found that the phenotypic response to stress was the same, but the induced and suppressed gene pools were different [27]. However, there were differences in the microflora genera between the treatment group and the control group in this study, especially with Rhodobacteraceae, Flavobacteriaceae, Haloferula, and nitrite bacteria. In addition, Lactobacillus was detected only in the water samples from the outlet of the copper ion generator. Therefore, correlating phenotypic and genotypic responses will provide new insights on how bacteria respond to a changing environment in the future.

\section{Conclusion}

There was no significant enrichment of $B$. rerio when the copper ion concentration was $0.15 \mathrm{mg} / \mathrm{L}$. In addition, microbiological analysis clearly showed that bacterial species richness and community diversity differed between the two groups. However, there was no sharp decrease in the microflora near the outlet of the copper ion generator. The relative abundances of Erythrobacter, nitrite bacteria, and Flavanobacteria were clearly higher in the treatment group than in the control group. Therefore, copper ion sterilization can be considered a relatively mild cleaning technology. Copper ion sterilization may greatly improve the abundance of beneficial bacteria, which may help control the organic matter and inorganic nitrogen pollution in RASs. These functional bacteria could be isolated and developed into bacterial agents for use in RASs in the near future. These bacterial agents have important prospective applications in the optimization of aquaculture processes, the improvement of aquaculture production, and the effective control of aquaculture disease risk. Incorporating this knowledge would provide opportunities to develop new system operations and could move system optimization boundary set the bound by current models. This would be expected to become popular in the aquaculture industry.

Supplementary Information The online version contains supplementary material available at https://doi.org/10.1007/s00284-021-02707-2.
Acknowledgements This work was supported by funds from the Central Public-interest Scientific Institution Basal Research Fund, CAFS (2020TD78). We thank LetPub (www.letpub.com) for its linguistic assistance during the preparation of this manuscript.

Author Contributions All authors contributed to the study conception and design. Material preparation was performed by SC and CG and data collection and analysis were performed by JS, XT and CG. The first draft of the manuscript was written by JS and XT. YZ and CZ contributed to the comments for manuscript edits. All authors commented on and read, consented to participate, consented to publication, and approved the final manuscript.

Data Availability Data are available from the corresponding authors upon reasonable request.

\section{Declarations}

Conflict of interest The authors declare that they have no conflicts of interest. This study was approved by the Animal Care and Use Committee of the Chinese Academy of Fishery Sciences, Beijing, China. All of the animal procedures were conducted in compliance with the Guidelines for the Care and Use of Laboratory Animals in China.

Open Access This article is licensed under a Creative Commons Attribution 4.0 International License, which permits use, sharing, adaptation, distribution and reproduction in any medium or format, as long as you give appropriate credit to the original author(s) and the source, provide a link to the Creative Commons licence, and indicate if changes were made. The images or other third party material in this article are included in the article's Creative Commons licence, unless indicated otherwise in a credit line to the material. If material is not included in the article's Creative Commons licence and your intended use is not permitted by statutory regulation or exceeds the permitted use, you will need to obtain permission directly from the copyright holder. To view a copy of this licence, visit http://creativecommons.org/licenses/by/4.0/.

\section{References}

1. Tian WT (2011) Application of modern biotechnology in aquaculture. Modern Anim Husband Sci Technol 5:269. https://doi. org/10.3969/j.issn.1673-1921.2011.05.263

2. Guillen J, Asche F, Carvalho N, Fernandez Polanco JM, Llorente I, Nielsen R, Nielsen M, Villasante S (2019) Aquaculture subsidies in the european union: evolution, impact and future potential for growth. Mar Policy 104:19-28. https://doi.org/10.1016/j.marpol.2019.02.045

3. Tang YT, Zhao LJ, Yang Y, Liu QG, Fan YC, Wu JY (2015) Effects of Hyriopsis cumingii, Hypophthalmichthys molitrix and Aristichthys nobilis polycultured on microbial community structure in aquaculture ponds /enclosures: a comparative study-Based on 16S rDNA sequences. Freshw Fish 45(03):70-77. https://doi. org/10.13721/j.cnki.dsyy.20150309.005

4. Amann RI (1995) Phylogenetic identifieation and in situ detection of individual microbial cells without cultivation. Microbiol Rev 59:143-169. https://doi.org/10.1016/S0882-4010(95)90076-4

5. Zhou T, Zheng XF, Tang JY, Qin JG, Wang Y (2017) Effect of three commercial microbial products on bacterial community in a freshwater fish polyculture system. Aquac Res 48(8):4449-4460

6. Mente E, Pierce GJ, Begoña Santos M, Neofitou C (2006) Effect of feed and feeding in the culture of salmonids on the marine 
aquatic environment: a synthesis for European aquaculture. Aquacult Int 14:499-522

7. Shan JJ, Guan CW, Zhang CL, Zhang YL, Wu F (2019) The Concentration of $\mathrm{Cu}^{2+}$ : effects on germicidal efficacy in recirculating aquaculture system. Chin Agric Sci Bull 35(9):138-142

8. Liu L (2017) Application of beneficial microorganisms in aquaculture. Jiangxi Agic 01:106-107

9. Yuan X, Fan QH (2015) Application of beneficial microorganisms in aquaculture. Sci Fish Farm 03(86 \& 04):86. https://doi.org/10. 14184/j.cnki.issn1004-843x.2015.03.054

10. Lang ZC, Zhou MH, Zhang QZ, Yin XY, Li YW (2020) Comprehensive treatment of marine aquaculture wastewater by a costeffective flow-through electro-oxidation process. Sci Total Environ 722:137812. https://doi.org/10.1016/j.scitotenv.2020.137812

11. Yuan LD, Li LZ, Tian XQ, Tang YY, Fan CQ, Huang HL, Yang Q (2016) Biodiversity and phylogenetic analysis of the gut microbiome of Euphausia superba Dana from the Rose Sea of the Antarctic Ocean. Adv Polar Sci 27(01):48-55. https://doi.org/10.13679/j. advps.2016.1.00048

12. De Schryver P, Vadstein O (2014) Ecological theory as a foundation to control pathogenic invasion in aquaculture. Int Soc Microb Ecol 8:2360-2368. https://doi.org/10.1038/ismej.2014.84

13. Bartelme RP, McLellan SL, Newton RJ (2017) Freshwater recirculating aquaculture system operations drive biofilter bacterial community shifts around a stable nitrifying consortium of ammonia-oxidizing archaea and Comammox Nitrospira. Front Microbiol. https://doi.org/10.3389/fmicb.2017.00101

14. Simon M, Scheuner C, Meier-Kolthoff JP, Brinkhoff T, WagnerDöbler I, Ulbrich M, Klenk HP, Schomburg D, Petersen J, Göker M (2017) Phylogenomics of Rhodobacteraceae reveals evolutionary adaptation to marine and non-marine habitats. Int Soc Microb Ecol 11:1483-1499. https://doi.org/10.1038/ismej.2016.198

15. Zhang HG, Ma SS, Li QF, Fu XJ, Zhang Y, Qu KM (2011) Analysis of the changes of microbial community structure on bio-carrier of Recirculating Aquaculture Systems (RAS). Environ Sci 32(01):231-239. https://doi.org/10.13227/j.hjkx.2011.01.031

16. Jooste PJ, CeliaJ H (1999) The taxonomy, ecology and cultivation of bacterial genera belonging to the family flavobacteriaceae. Int J Food Microbiol 53:81-94. https://doi.org/10.1016/s01681605(99)00162-2

17. Avendao-Herrera R, Benavides I, Espina JA, Soto-Comte D, Poblete-Morales M, Valdés JA, Feijóo CG, Reyes AE (2020) Zebrafish (danio rerio) as an animal model for bath infection by flavobacterium psychrophilum. J Fish Dis 43(5):561-570. https:// doi.org/10.1111/jfd.13156

18. Orso S, Gouy M, Navarro E, Normand P (1994) Molecular phylogenetic analysis of Nitrobacter spp. Int J Syst Bacteriol 44(1):8386. https://doi.org/10.1099/00207713-44-1-83
19. Yin J, Xu WF (2009) Ammonia biofiltration and community analysis of ammonia-oxidizing bacteria in biofilters. Biores Technol 100(17):3869-3876. https://doi.org/10.1016/j.biortech.2009.03. 021

20. Purkhold U, Pommerening-Röser A, Juretschko S, Schmid MC, Koops HP, Wagner M (2000) Phylogeny of all recognized species of ammonia oxidizers based on comparative 16S rRNA and amoA sequence analysis: implications for molecular diversity surveys. Appl Environ Microbiol 66(12):5368-5382. https://doi.org/10. 1128/AEM.66.12.5368-5382.2000

21. J1 Z, Jiang M, Wang CF, Tong XL, Jin J, Gu DP, Hu WG, Yu ZL, Dai XL (2017) Microbial diversity in bio-filter of the recirculating aquaculture system for Litopenaeus vannamei. Progress Fish Sci 38(05):73-82. https://doi.org/10.11758/yykxjz.20160330001

22. Chen Q, Li GY, Luo K, Kong J, Mo ZL, Luan S, Li J, Cao BX, Zhang YL (2017) Microbial diversity in broodstock waters of the two genders of Litopenaeus Vannamel. Oceanol Limnol Sin 48(1):130-138. https://doi.org/10.11693/hyhz20160700176

23. Guan ZG, Dou Y, Yu SG, Zhou WL, Shao P, Gao JW, Jia XY (2019) Effects of growth and antioxidase activities of Litopenaeus vanname $i$ through rhizobium adding in the fodder. Tianjin Agric Sci 25(07):47-51. https://doi.org/10.3969/j.issn.1006-6500.2019. 07.010

24. Rowan NJ (2011) Defining established and emerging microbial risks in the aquatic environment: current knowledge, implications, and outlooks. Int J Microbiol. https://doi.org/10.1155/2011/ 462832

25. Luo X, Yuan LX, Zhang WL, Zhong WZ, Jiang YF, Zhang Y, Xu DS (2019) Correlation study between resistance genes and microbial communities in pharmaceutical wastewater treatment plants. China Environ Sci 39(2):831-838. https://doi.org/10.19674/j.cnki. issn1000-6923.2019.0102

26. Cangelosi GA, Meschke JS (2014) Dead or Alive: Molecular Assessment of Microbial Viability. Appl Environ Microbiol 80(19):5884-5891. https://doi.org/10.1128/aem.01763-14

27. del Muñoz M, Benomar N, Lerma LL, Knapp CW, Gálvez A, Abriouel H (2016) Biocide tolerance, phenotypic and molecular response of lactic acid bacteria isolated from naturally-fermented Aloreña table to different Physico-chemical stresses. Food Microbiol 60:1-12

Publisher's Note Springer Nature remains neutral with regard to jurisdictional claims in published maps and institutional affiliations. 